\title{
Análise da Composição do solo e sua Influência no Processo Corrosivo de Postes Metálicos na UFERSA, Campus Caraúbas-RN.
}

\author{
Anderson Nunes Silva* (Graduando em Ciência e Tecnologia na Universidade Federal Rural do Semi-Árido - \\ UFERSA, Campus Caraúbas-RN.) \\ Anderson Milano de Sousa Fernandes (Graduando em Ciência e Tecnologia na Universidade Federal Rural do \\ Semi-Árido - UFERSA, Campus Caraúbas-RN.) \\ André Moreira de Oliveira (Docente do curso de Ciência e Tecnologia na Universidade Federal Rural do Semi- \\ Árido - UFERSA, Campus Caraúbas-RN.) \\ Daniel Freitas Freire Martins (Docente do curso de Ciência e Tecnologia na Universidade Federal Rural do \\ Semi-Árido - UFERSA, Campus Caraúbas-RN.) \\ *E-mail: andersonunes.luc@hotmail.com
}

\begin{abstract}
Resumo:
Dentre os materiais mais utilizados na construção e montagens de postes e torres destacam-se os metais, que quando expostos ao meio ambiente estão sujeitos à corrosão. A corrosão pode ser definida como um processo de deterioração de materiais metálicos e não metálicos por influência de agentes físico-químicos ligados ou não a esforços mecânicos. Os agentes que influenciam no processo corrosivo são: solo, temperatura, poluição, umidade e gases. O solo possui um grande número de variáveis que contribuem para o aumento do processo corrosivo em estruturas metálicas, algumas foram estudadas nesse trabalho, seguindo o manual de métodos de analise do solo da Empresa Brasileira de Pesquisa Agropecuária (EMBRAPA). Os resultados foram baseados de acordo com as analises físico-química da umidade, condutividade, $\mathrm{pH}$, matéria orgânica, magnésio, sódio e cálcio. Sendo possível com esses resultados caracterizar os solos segundo sua salinidade, mostrando que os solos estudados são do tipo salino. Faz-se necessário este trabalho para um estudo sobre a influência da composição físico-química do solo no processo corrosivo dos postes de iluminação da UFERSACampus Caraúbas-RN, buscando propor possíveis técnicas de prevenção do processo corrosivo, como a proteção catódica por metal de sacrifício e o revestimento com tintas anticorrosivas.
\end{abstract}

Palavrras-chave: Corrosão; estruturas metálicas; solo; analises físico-química; técnicas de prevenção.

Espaço reservado para organização do congresso. 


\section{I NTRODUÇÃO}

Entre os materiais mais utilizados na construção e montagens de postes e torres destacam-se os metais, devido sua resistência mecânica, por serem moldáveis e por alguns apresentarem melhor custodurabilidade do que outros materiais. Entretanto, os metais expostos ao meio ambiente estão sujeitos à corrosão.

Segundo Gentil (2011), num aspecto muito difundido e aceito universalmente pode-se definir corrosão como a deterioração de um material, geralmente metálico, por ação química ou eletroquímica do meio ambiente aliado ou não a esforços mecânicos. A deterioração causada pela interação físicoquímica entre o material e o seu meio operacional representa alterações prejudiciais indesejáveis, sofridas pelo material, tais como desgaste, variações químicas ou modificações estruturais, tornando-o inadequado para o uso.

A corrosão ocorre devido aos fenômenos da oxidação, que consiste na perda de elétrons por uma espécie química, e da redução, que é o ganho de elétrons por uma espécie química. Assim, os processos corrosivos são considerados reações químicas heterogêneas ou reações eletroquímicas que se passam geralmente na superfície de separação entre o metal e o meio corrosivo (AMARAL, 2008).

Os meios corrosivos mais frequentes são: atmosfera, águas naturais, solo, e produtos químicos (DUARTE, 2003).

Por sua vez, a corrosão de materiais em contato com solo é um fenômeno bastante complexo em que são envolvidas múltiplas variáveis. Comparado à corrosão atmosférica e a outros tipos de corrosão, esta ainda é um assunto menos investigado, dado a complexidade do solo como meio corrosivo (NOBREGA, 2003).

O solo é uma coleção de corpos naturais constituídos por partes sólidas, líquidas e gasosas, tridimensionais, dinâmicos, que são formados por materiais minerais e orgânicos que ocupam a maior parte do manto superficial das extensões continentais do planeta (EMBRAPA, 1997).

São diversos os fatores que contribuem para a corrosividade dos solos, dentre estes se incluem: o tipo de solo, suas características estruturais, texturais (composição granulométrica), permeabilidade, teor de umidade, posição do nível do lençol freático, grau de aeração, conteúdo de sais solúveis, acidez, presença de micro-organismos, etc. Para tornar o problema ainda mais difícil, alguns destes fatores são inter-relacionados (NOBREGA, 2003).

A agressividade do solo aos metais engloba dois tipos: a agressividade específica e a agressividade relativa. A primeira se relaciona ao amplo elenco de propriedades físico-químicas e biológicas do solo, enquanto a segunda apresenta-se dependente de fatores externos, capazes de modificar o processo de corrosão, como, por exemplo, a presença de correntes de fuga (MAGALHÃES, 2002).

O processo corrosivo é um grande problema em diversos setores, tais como: construção civil, mecânica, química, aeronáutica e telecomunicações. A problematização da corrosão tem como principais intuitos minimizar os gastos nestes setores, no reparo de peças ou trocas das que estão deterioradas, além de garantir a segurança do homem, evitando acidentes provenientes deste processo.

Países industrializados como os EUA apresentam prejuízos da ordem de 300 bilhões de dólares anuais, superando catástrofes como enchentes e fogo naquele mesmo país. Com relação ao Brasil, apesar de não existirem estudos completos sobre os prejuízos causados pela corrosão, é possível fazerse uma estimativa, onde o custo da corrosão para um país industrializado é de aproximadamente 3,5\% do seu PIB. Estima-se que no Brasil os prejuízos decorrentes da corrosão ultrapassem 30 bilhões de dólares anuais (BRUSAMARELLO et al, 2002).

Desta forma, este trabalho tem como objetivo fazer um estudo sobre a influência da composição físico-química do solo no processo corrosivo dos postes de iluminação da UFERSACampus Caraúbas-RN, buscando propor possíveis técnicas para tardar e prevenir o processo corrosivo, possibilitando futuramente a economia com os custos que seriam destinados ao reparo ou substituições de peças dos postes deteriorados pela corrosão. 


\section{METODOLOGIA}

Para análise da influência do solo no processo corrosivo da base e parafusos de postes de ferro, foram feitas análises físico-químicas, com amostras de $20 \mathrm{~cm}$ de profundidade do solo onde se encontram os postes. Sendo o estudo feito em 10 pontos (10 postes) os quais foram selecionados buscando a maior proporção possível da área que contem postes na UFERSA-Campus Caraúbas-RN. As amostras do solo foram coletadas de 30 em 30 dias.

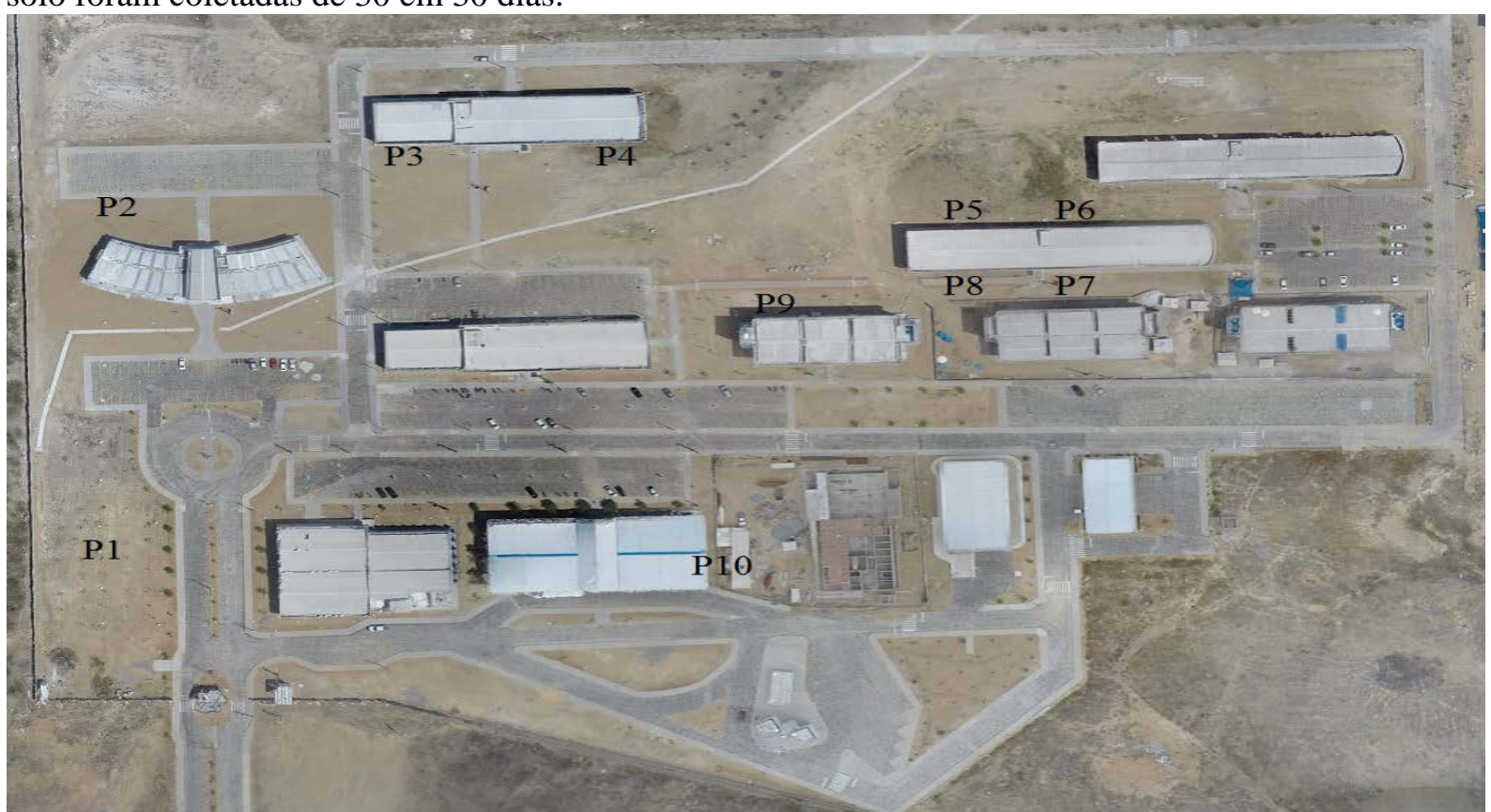

Figura 01: Imagem aérea ortorretificada da UFERSA Campus Caraúbas através de veículo aéreo não tripulado (VANT), com os pontos de coletas do solo marcados. Fonte: Adaptada de PEIXOTO (2015).

Os princípios e procedimentos para preparação e análises físico-química seguiram o manual de métodos de analise do solo da Empresa Brasileira de Pesquisa Agropecuária (EMBRAPA) e estão descritos sumariamente a seguir:

\subsection{Coleta e preparação das amostras}

As amostras foram coletadas com um trado holandês, sempre com profundidade de $20 \mathrm{~cm}$. Depois armazenadas em sacos plásticos enumerados de 1 a 10, posteriormente foram levadas para o laboratório de química da Universidade Federal Rural do Semi-Árido, Campus de Caraúbas, onde foram secadas, destorroadas, separadas nas frações do solo por tamisação e homogeneização da fração $<2 \mathrm{~mm}$, assim denominada "terra fina seca ao ar" (TFSA).

\subsection{Umidade}

Ao chegar no laboratório, aproximadamente 50,0 gramas das amostras solo de cada ponto foram pesadas, em triplicata, em um béquer numerado, previamente lavado, seco e pesado, depois levadas para estufa a $105^{\circ} \mathrm{C}$ e deixadas nessas condições por 24 horas. Em seguida, foi retirado da estufa e colocado no dessecador. Após atingir a temperatura ambiente, o mesmo foi pesado e a sua massa utilizada para calcular o teor de umidade a partir da equação 01 :

$$
\begin{aligned}
& 100(\mathrm{a}-\mathrm{b}) / \mathrm{a} \\
& \mathrm{a}=\text { peso da amostra úmida }(\mathrm{g}) \\
& \mathrm{b}=\text { peso da amostra seca }(\mathrm{g})
\end{aligned}
$$




\subsection{Condutividade}

Foi colocado $10 \mathrm{~cm}^{3}$ de solo em copos plásticos enumerados. Em seguida, colocou-se $25 \mathrm{ml}$ de água destilada em cada copo, homogeneizou-se com bastão de vidro, deixou-se em repouso por uma hora e, finalmente, mediu-se a condutividade por meio de um condutivímetro, marca HANNA instrumentsmodelo DIST WP, com o eletrodo imerso na suspensão solo líquido.

\section{4. $\mathrm{pH}$}

Foi colocado $10 \mathrm{~cm}^{3}$ de solo em copos plásticos enumerados. Em seguida, colocou-se $25 \mathrm{ml}$ de água destilada em cada copo, homogeneizou-se com bastão de vidro e deixou-se em repouso por uma hora. Depois homogeneizou-se novamente com bastão de vidro as amostras e começou-se a medição, por meio de um pHmetro, marca TECNAL- modelo Tec-3MP, com o eletrodo imerso na suspensão solo líquido.

\subsection{Matéria Orgânica}

Colocou-se aproximadamente $5 \mathrm{~g}$ de solo em cadinhos numerados, previamente pesados, e levou-se para a mufla a $500^{\circ} \mathrm{C}$, deixando por 3 horas. Em seguida, colocou-se as amostras no dessecador e após atingir temperatura ambiente, pesou-se novamente e, através da Equação 02 encontrou-se o valor da matéria orgânica.

$$
\begin{gathered}
100(\mathrm{a}-\mathrm{b}) / \mathrm{a} \\
\mathrm{a}=\text { peso da amostra seca }(\mathrm{g}) \\
\mathrm{b}=\text { peso da amostra calcinada }(\mathrm{g})
\end{gathered}
$$

\subsection{Extração com Solução de Mehlich 1}

A solução extratora de Mehlich 1, também chamada de solução duplo-ácida ou de Carolina do Norte, é constituída por uma mistura de HCI $0,05 \mathrm{M}+\mathrm{H}_{2} \mathrm{SO}_{4}$ 0,0125M. Para fazer a analises colocou-se 10 $\mathrm{cm}^{3}$ de TFSA em erlenmeyer de $125 \mathrm{ml}$ com 100ml da solução de Mehlich1, agitou-se por 5 minutos, e deixou-se em repouso por 12 horas. Por ultimo, retirou-se a solução dos erlenmeyes e colocou-se em recipientes numerados, e encaminhou-se para a medição de sódio no fotômetro de chama, marca TECNOW- modelo 7000.

\subsection{Determinação do Cálcio e Magnésio}

Pesou-se $10 \mathrm{~cm}^{3}$ de solo, colocou-se em erlenmeyer de $250 \mathrm{ml}$ e adicionou-se $100 \mathrm{ml}$ de solução de $\mathrm{KCl}$ $1,0 \mathrm{M}$, agitou-se por 5 minutos e pipetou-se o sobrenadante para erlenmeyer de $200 \mathrm{~mL}$ para determinação do cálcio + magnésio e cálcio trocáveis. Posteriormente transferiu-se $25 \mathrm{~mL}$ do extrato para um erlenmeyer de $250 \mathrm{ml}$, adicionou-se $4 \mathrm{~mL}$ do coquetel tampão e uma pitada do indicador Negro de eriocromo T e titulou-se, imediatamente, com a solução de EDTA 0,0125 M, até viragem da cor rósea para azul puro ou esverdeada. A determinação do cálcio foi feita quando transferiu-se $25 \mathrm{~mL}$ do extrato para um erlenmeyer de $250 \mathrm{~mL}$, colocou-se $2 \mathrm{ml}$ de trietanolamina a $50 \%$, $2 \mathrm{ml}$ de $\mathrm{KOH}$ a 10\% e uma pitada de Calcon. Titulou-se com solução de EDTA 0,0125 M até viragem da cor rósea

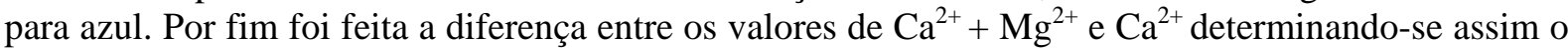
valor de magnésio presente.

\section{RESULTADOS E DISCUSSÃO}

Os resultados das análises físico-químicas referentes aos solos estudados estão na tabela 01. São apresentados os valores de umidade (\%), condutividade $(\mu \mathrm{s} / \mathrm{cm}), \mathrm{pH}$, matéria orgânica, magnésio ( $\mathrm{cmol} / \mathrm{L})$, sódio $(\mathrm{cmol} / \mathrm{L})$ e cálcio $(\mathrm{cmol} / \mathrm{L})$. 
Tabela 01- Resultado das análises físico-químicas.

\begin{tabular}{|c|c|c|c|c|c|c|}
\hline \multirow{10}{*}{$\begin{array}{l}\text { Umidade } \\
(\%)\end{array}$} & Coletas & P1 & P2 & P3 & P4 & P5 \\
\hline & $1^{\mathrm{a}}$ & $3,97 \pm 0,0627$ & $11,08 \pm 0,5574$ & $9,16 \pm 0,1569$ & $18,35 \pm 1,2855$ & $7,16 \pm 0,2856$ \\
\hline & $2^{a}$ & $0,60 \pm 0,0302$ & $3,49 \pm 0,8061$ & $5,47 \pm 0,1168$ & $16,01 \pm 0,1084$ & $6,27 \pm 0,4849$ \\
\hline & $3^{a}$ & $0,42 \pm 0,0395$ & $2,74 \pm 0,0939$ & $6,86 \pm 0,2234$ & $12,75 \pm 0,6126$ & $4,86 \pm 0,3387$ \\
\hline & $4^{a}$ & $0,35 \pm 0,0083$ & $3,89 \pm 0,4248$ & $4,82 \pm 0,0626$ & $11,03 \pm 1,0823$ & $4,22 \pm 0,8857$ \\
\hline & Coletas & P6 & P7 & P8 & P9 & P10 \\
\hline & $1^{\mathrm{a}}$ & $5,88 \pm 0,0676$ & $5,67 \pm 0,3196$ & $5,21 \pm 0,155$ & $3,27 \pm 0,0647$ & $8,95 \pm 0,6020$ \\
\hline & $2^{a}$ & $5,24 \pm 0,1657$ & $4,80 \pm 0,6482$ & $4,02 \pm 0,1194$ & $3,90 \pm 0,0970$ & $8,14 \pm 0,3530$ \\
\hline & $3^{\mathrm{a}}$ & $4,09 \pm 0,0656$ & $2,83 \pm 0,0791$ & $2,80 \pm 0,1576$ & $3,42 \pm 0,0554$ & $7,78 \pm 0,4986$ \\
\hline & $4^{\mathrm{a}}$ & $4,07 \pm 0,1411$ & $3,20 \pm 0,2129$ & $2,43 \pm 0,3955$ & $2,57 \pm 0,1875$ & $4,95 \pm 0,0089$ \\
\hline \multirow{10}{*}{$\begin{array}{l}\text { Condutividade } \\
(\mu \mathrm{s} / \mathrm{cm})\end{array}$} & Coletas & P1 & P2 & P3 & P4 & P5 \\
\hline & $1^{\mathrm{a}}$ & $73,01 \pm 4,42$ & $273,20 \pm 5,09$ & $248,20 \pm 1,98$ & $171,10 \pm 14,04$ & $176,50 \pm 9,53$ \\
\hline & $2^{a}$ & $112,9 \pm 9,64$ & $60,48 \pm 2,33$ & $135,23 \pm 7,46$ & $123,9 \pm 3,8$ & $85,28 \pm 6,34$ \\
\hline & $3^{a}$ & $304,95 \pm 16,9$ & $100,86 \pm 2,33$ & $156,17 \pm 1,66$ & $123,43 \pm 2,06$ & $195,00 \pm 6,71$ \\
\hline & $4^{a}$ & $223,90 \pm 8,63$ & $76,29 \pm 3,22$ & $68,99 \pm 2,50$ & $111,97 \pm 12,80$ & $220,03 \pm 16,0$ \\
\hline & Coletas & P6 & P7 & P8 & P9 & P10 \\
\hline & $1^{\mathrm{a}}$ & $308,87 \pm 8,90$ & $77,25 \pm 0,81$ & $109,4 \pm 3,96$ & $947,67 \pm 14,04$ & $190,73 \pm 3,1$ \\
\hline & $2^{\mathrm{a}}$ & $77,59 \pm 3,91$ & $60,90 \pm 1,99$ & $55,32 \pm 4,64$ & $280,43 \pm 3,8$ & $86,75 \pm 6,01$ \\
\hline & $3^{\mathrm{a}}$ & $127,03 \pm 6,05$ & $81,91 \pm 6,27$ & $283,15 \pm 11,1$ & $300,13 \pm 2,06$ & $104,08 \pm 5,07$ \\
\hline & $4^{\mathrm{a}}$ & $199,40 \pm 4,67$ & $100,37 \pm 3,20$ & $60,94 \pm 3,84$ & $443,94 \pm 12,8$ & $83,23 \pm 6,79$ \\
\hline \multirow{10}{*}{$\mathrm{pH}$} & Coletas & P1 & P2 & P3 & P4 & P5 \\
\hline & $1^{\mathrm{a}}$ & $6,14 \pm 0,16$ & $7,96 \pm 0,13$ & $8,21 \pm 0,04$ & $8,10 \pm 0,00$ & $8,11 \pm 0,01$ \\
\hline & $2^{a}$ & $6,84 \pm 0,09$ & $7,76 \pm 0,06$ & $8,35 \pm 0,17$ & $8,41 \pm 0,25$ & $8,21 \pm 0,04$ \\
\hline & $3^{\mathrm{a}}$ & $5,78 \pm 0,16$ & $7,61 \pm 0,20$ & $8,54 \pm 0,19$ & $9,06 \pm 0,06$ & $8,62 \pm 0,06$ \\
\hline & $4^{\mathrm{a}}$ & $5,68 \pm 0,08$ & $8,06 \pm 0,46$ & $8,70 \pm 0,05$ & $8,72 \pm 0,02$ & $8,51 \pm 0,03$ \\
\hline & Coletas & P6 & P7 & P8 & P9 & P10 \\
\hline & $1^{\mathrm{a}}$ & $7,93 \pm 0,02$ & $7,76 \pm 0,05$ & $7,80 \pm 0,05$ & $7,25 \pm 0,09$ & $7,25 \pm 0,10$ \\
\hline & $2^{\mathrm{a}}$ & $7,84 \pm 0,18$ & $7,61 \pm 0,06$ & $7,86 \pm 0,20$ & $6,79 \pm 0,08$ & $8,17 \pm 0,30$ \\
\hline & $3^{\mathrm{a}}$ & $8,46 \pm 0,07$ & $8,48 \pm 0,04$ & $7,63 \pm 0,13$ & $7,32 \pm 0,12$ & $7,79 \pm 0,03$ \\
\hline & $4^{\mathrm{a}}$ & $8,37 \pm 0,03$ & $7,66 \pm 0,08$ & $8,34 \pm 0,20$ & $6,79 \pm 0,08$ & $8,17 \pm 0,30$ \\
\hline \multirow{10}{*}{$\begin{array}{c}\text { Matéria } \\
\text { Orgânica } \\
(\%)\end{array}$} & Coletas & P1 & P2 & P3 & P4 & P5 \\
\hline & $1^{\mathrm{a}}$ & $1,23 \pm 0,067$ & $3,64 \pm 0,207$ & $1,12 \pm 0,014$ & $1,039 \pm 0,035$ & $1,28 \pm 0,013$ \\
\hline & $2^{\mathrm{a}}$ & $0,88 \pm 0,155$ & $0,51 \pm 0,0332$ & $0,88 \pm 0,065$ & $2,84 \pm 0,416$ & $2,67 \pm 0,062$ \\
\hline & $3^{\mathrm{a}}$ & $1,09 \pm 0,013$ & $1,75 \pm 0,083$ & $3,12 \pm 0,087$ & $3,576 \pm 0,036$ & $3,06 \pm 0,077$ \\
\hline & $4^{\mathrm{a}}$ & $1,44 \pm 0,025$ & $1,81 \pm 0,164$ & $2,42 \pm 0,267$ & $3,693 \pm 0,243$ & $2,25 \pm 0,125$ \\
\hline & Coletas & P6 & P7 & P8 & P9 & P10 \\
\hline & $1^{\mathrm{a}}$ & $2,19 \pm 0,071$ & $1,66 \pm 0,120$ & $1,51 \pm 0,072$ & $1,82 \pm 0,045$ & $2,14 \pm 0,430$ \\
\hline & $2^{\mathrm{a}}$ & $3,04 \pm 0,068$ & $2,20 \pm 0,227$ & $1,23 \pm 0,021$ & $1,82 \pm 0,116$ & $5,10 \pm 0,079$ \\
\hline & $3^{\mathrm{a}}$ & $3,12 \pm 0,039$ & $1,32 \pm 0,048$ & $1,70 \pm 0,258$ & $0,30 \pm 0,013$ & $0,50 \pm 0,089$ \\
\hline & $4^{\mathrm{a}}$ & $2,54 \pm 0,276$ & $1,29 \pm 0,143$ & $0,99 \pm 0,054$ & $1,67 \pm 0,083$ & $1,72 \pm 0,070$ \\
\hline \multirow{10}{*}{$\begin{array}{l}\text { Magnésio } \\
\text { (cmol/L) }\end{array}$} & Coletas & P1 & $\mathrm{P} 2$ & P3 & $\mathrm{P} 4$ & P5 \\
\hline & $1^{\mathrm{a}}$ & $1,40 \pm 0,10$ & $7,17 \pm 0,12$ & $2,03 \pm 0,21$ & $2,23 \pm 0,06$ & $3,20 \pm 0,10$ \\
\hline & $2^{\mathrm{a}}$ & $1,67 \pm 0,06$ & $1,70 \pm 0,87$ & $1,03 \pm 0,06$ & $1,57 \pm 0,06$ & $1,63 \pm 0,25$ \\
\hline & $3^{\mathrm{a}}$ & $1,57 \pm 0,06$ & $1,27 \pm 0,06$ & $0,90 \pm 0,26$ & $0,25 \pm 0,07$ & $1,20 \pm 0,66$ \\
\hline & $4^{\mathrm{a}}$ & $2,43 \pm 0,21$ & $1,57 \pm 0,32$ & $0,83 \pm 0,29$ & $1,17 \pm 0,40$ & $1,40 \pm 0,82$ \\
\hline & Coletas & P6 & P7 & P8 & P9 & P10 \\
\hline & $1^{\mathrm{a}}$ & $2,53 \pm 0,06$ & $1,90 \pm 0,40$ & $1,63 \pm 0,23$ & $1,60 \pm 0,17$ & $2,07 \pm 0,21$ \\
\hline & $2^{\mathrm{a}}$ & $1,63 \pm 0,23$ & $1,10 \pm 0,20$ & $1,77 \pm 0,31$ & $1,37 \pm 0,20$ & $2,97 \pm 0,38$ \\
\hline & $3^{\mathrm{a}}$ & $2,37 \pm 0,38$ & $0,97 \pm 0,21$ & $1,33 \pm 0,15$ & $0,83 \pm 0,15$ & $2,43 \pm 0,45$ \\
\hline & $4^{\mathrm{a}}$ & $1,13 \pm 0,12$ & $1,03 \pm 0,06$ & $1,40 \pm 0,92$ & $0,67 \pm 0,06$ & $2,20 \pm 0,62$ \\
\hline
\end{tabular}


Tabela 01- Resultado das análises físico-químicas.

\begin{tabular}{|c|c|c|c|c|c|c|}
\hline \multirow{10}{*}{$\begin{array}{c}\text { Cálcio } \\
\text { (cmol/L) }\end{array}$} & Coletas & P1 & P2 & P3 & P4 & P5 \\
\hline & $1^{\mathrm{a}}$ & $1,47 \pm 0,06$ & $8,63 \pm 0,12$ & $3,03 \pm 0,06$ & $3,80 \pm 0,00$ & $4,67 \pm 0,06$ \\
\hline & $2^{\mathrm{a}}$ & $1,80 \pm 0,00$ & $1,97 \pm 0,06$ & $1,63 \pm 0,06$ & $3,57 \pm 0,06$ & $1,87 \pm 0,06$ \\
\hline & $3^{\mathrm{a}}$ & $1,73 \pm 0,06$ & $4,80 \pm 0,00$ & $2,77 \pm 0,06$ & $4,27 \pm 0,59$ & $4,40 \pm 0,17$ \\
\hline & $4^{\mathrm{a}}$ & $3,76 \pm 0,56$ & $5,27 \pm 0,25$ & $2,97 \pm 0,71$ & $4,83 \pm 0,40$ & $5,70 \pm 0,17$ \\
\hline & Coletas & P6 & P7 & P8 & P9 & P10 \\
\hline & $1^{\mathrm{a}}$ & $5,37 \pm 0,06$ & $3,17 \pm 0,06$ & $4,00 \pm 0,00$ & $6,43 \pm 0,12$ & $5,87 \pm 0,15$ \\
\hline & $2^{\mathrm{a}}$ & $4,43 \pm 0,12$ & $3,60 \pm 0,10$ & $3,30 \pm 0,00$ & $4,10 \pm 0,10$ & $4,87 \pm 0,06$ \\
\hline & $3^{a}$ & $4,00 \pm 0,17$ & $3,83 \pm 0,46$ & $3,87 \pm 0,06$ & $4,40 \pm 0,36$ & $4,07 \pm 0,12$ \\
\hline & $4^{\mathrm{a}}$ & $6,23 \pm 0,06$ & $4,50 \pm 0,00$ & $4,23 \pm 0,40$ & $6,63 \pm 0,12$ & $5,27 \pm 0,40$ \\
\hline \multirow{10}{*}{$\begin{array}{c}\text { Sódio } \\
\text { (cmol/L) }\end{array}$} & Coletas & P1 & $\mathrm{P} 2$ & P3 & $\mathrm{P} 4$ & P5 \\
\hline & $1^{\mathrm{a}}$ & $0,30 \pm 0,00$ & $2,10 \pm 0,00$ & $1,53 \pm 0,06$ & $2,00 \pm 0,00$ & $0,30 \pm 0,00$ \\
\hline & $2^{a}$ & $0,30 \pm 0,00$ & $0,00 \pm 0,00$ & $0,60 \pm 0,00$ & $5,43 \pm 0,15$ & $0,10 \pm 0,00$ \\
\hline & $3^{a}$ & $0,06 \pm 0,00$ & $0,04 \pm 0,00$ & $1,00 \pm 0,00$ & $7,97 \pm 0,06$ & $0,50 \pm 0,00$ \\
\hline & $4^{\mathrm{a}}$ & $1,43 \pm 0,06$ & $0,40 \pm 0,00$ & $0,30 \pm 0,00$ & $5,53 \pm 0,06$ & $1,00 \pm 0,00$ \\
\hline & Coletas & P6 & P7 & P8 & P9 & P10 \\
\hline & $1^{\mathrm{a}}$ & $0,67 \pm 0,06$ & $0,10 \pm 0,00$ & $0,10 \pm 0,00$ & $0,20 \pm 0,00$ & $0,30 \pm 0,00$ \\
\hline & $2^{a}$ & $0,10 \pm 0,00$ & $0,00 \pm 0,00$ & $0,00 \pm 0,00$ & $0,00 \pm 0,00$ & $0,10 \pm 0,00$ \\
\hline & $3^{a}$ & $0,23 \pm 0,06$ & $0,10 \pm 0,00$ & $0,00 \pm 0,00$ & $0,00 \pm 0,00$ & $0,00 \pm 0,00$ \\
\hline & $4^{\mathrm{a}}$ & $0,40 \pm 0,00$ & $0,00 \pm 0,00$ & $0,00 \pm 0,00$ & $0,00 \pm 0,00$ & $0,10 \pm 0,00$ \\
\hline
\end{tabular}

A umidade do solo está relacionado a presença de água que há no mesmo. Os materiais metálicos em contato com a água tendem a sofrer corrosão, dependendo das impurezas que estão presentes na mesma, tais como: saís solúveis, gases dissolvidos, bactérias, matéria orgânica e sólidos suspensos (GENTIL, 2011). O gráfico 01 é referente à umidade relativa.

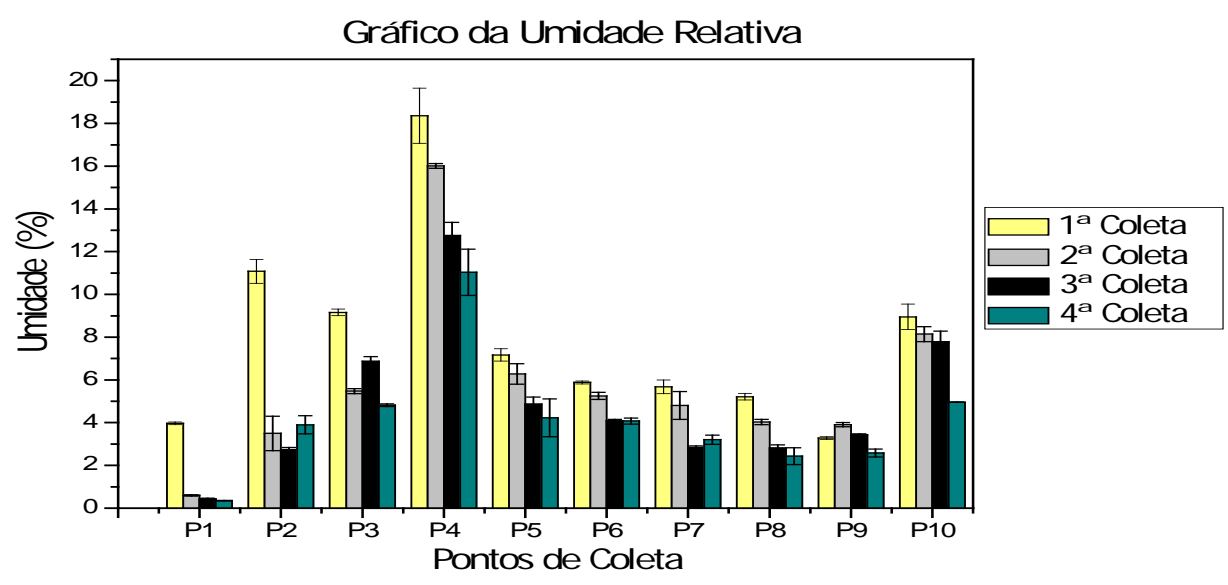

Gráfico 01: Gráfico da umidade relativa

O ponto 4 foi o que apresentou a maior umidade relativa chegando perto dos $20 \%$, em seguida o ponto 2 com quase $12 \%$, os outros pontos seguiram, praticamente, o mesmo padrão. A umidade vem decrescendo no decorrer das coletas mês a mês, provavelmente, devido ao período de chuvas diminuírem, na Microrregião da Chapada do Apodi e na Mesorregião Oeste Potiguar do Nordeste nesses meses do ano (Ver Figura 02). Como diz GENTIL (2011), em épocas de chuva, fatores como umidade e resistividade elétrica serão, completamente, diferentes daqueles verificados em épocas não chuvosas. 


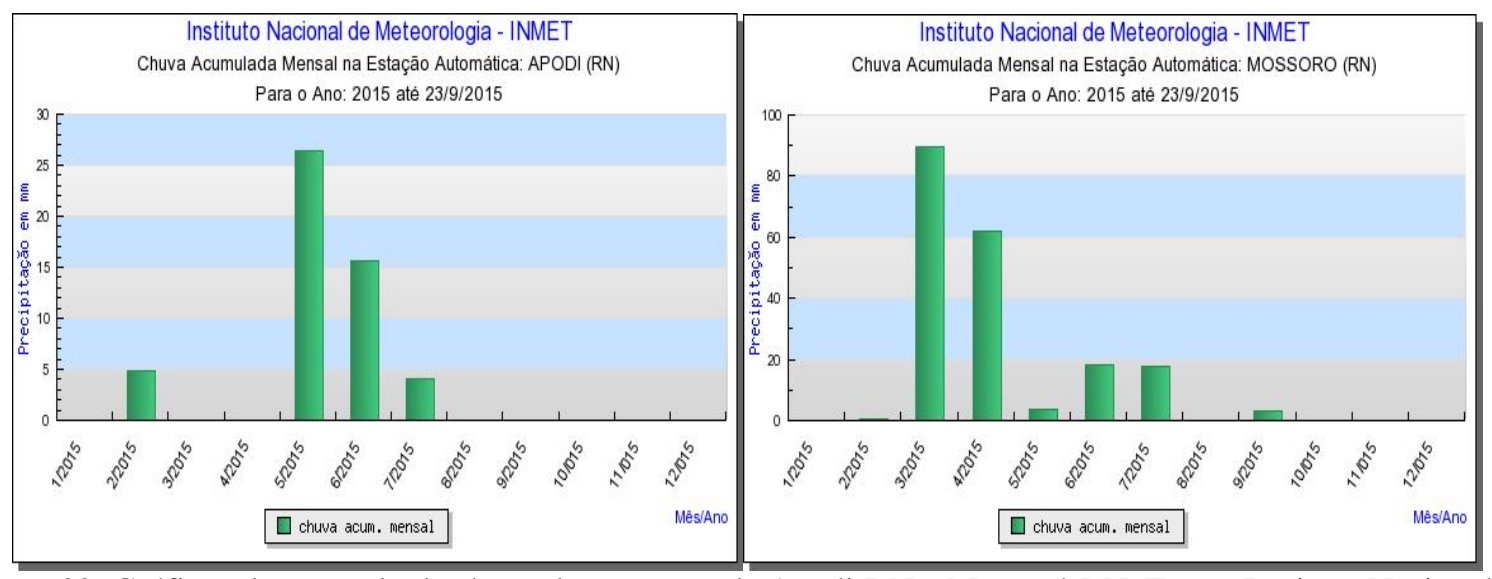

Figura 02: Gráficos do acumulo de chuva das estações de Apodi-RN e Mossoró-RN. Fonte: Instituto Nacional de Meteorologia - INMET.

A condutividade do solo está relacionada com a quantidade de sais existentes no mesmo, podendo verificar-se a condução da corrente elétrica. Quanto maior a condução da corrente elétrica mais fácil fica o processo eletroquímico da corrosão. O gráfico 02 é referente à condutividade dos solos estudados.

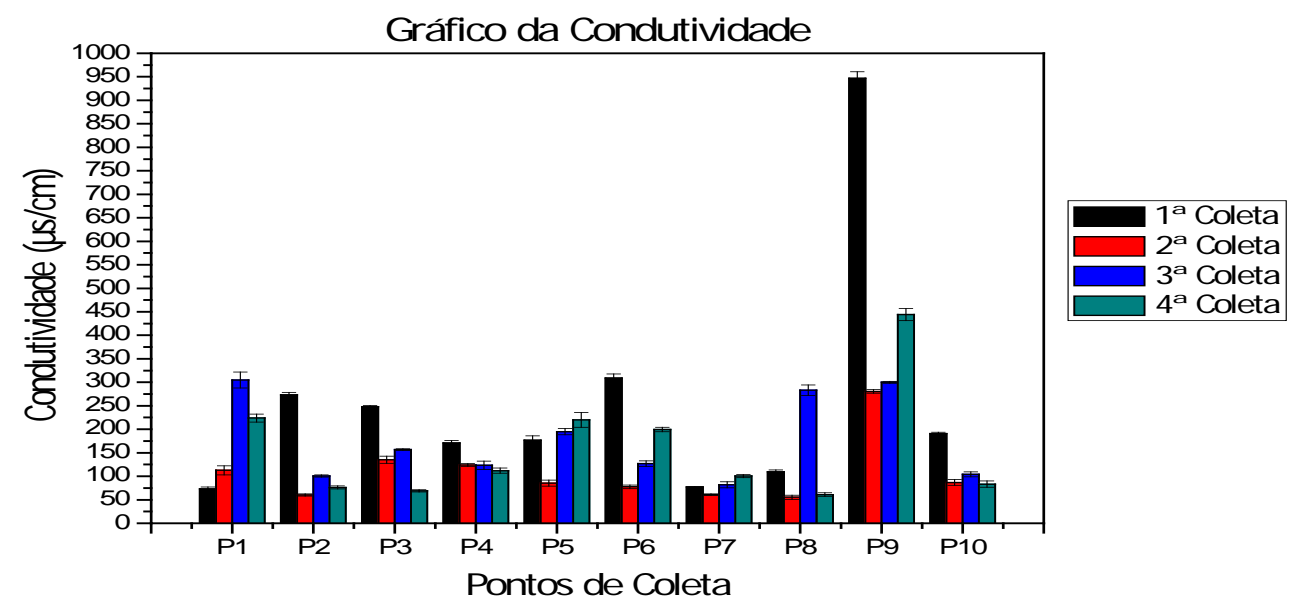

Gráfico 02: Condutividade.

O ponto 9 foi o que apresentou maiores valores de condutividade, os restantes seguiram praticamente o mesmo padrão. Observa-se que quanto maior o teor de umidade menor a condutividade nos pontos estudados, devido à solubilização dos sais e sua posterior lixiviação, causadas pelo fluxo de água. Comparando o gráfico 01 e gráfico 02, pode-se perceber que o ponto 4 que apresenta alto teor de umidade possui baixa condutividade, e o ponto 9 que possui baixa umidade já tem uma alta condutividade isso em todas as coletas.

$\mathrm{O} \mathrm{pH}$ do solo é um forte fator para o processo corrosivo dos metais, a acidez do solo vai influenciar no processo corrosivo dependendo de qual material esta inserido naquele meio, pois há metais que se corroem em meios ácidos (ferro, magnésio, zinco) e outros que se corroem no meio básico (alumínio, chumbo entre outros) (GENTIL, 2011). O material em questão neste trabalho são postes de ligas metálicas contendo ferro, e o ferro se oxida mais facilmente em meio ácido. O gráfico 03 representa os valores do $\mathrm{pH}$. 


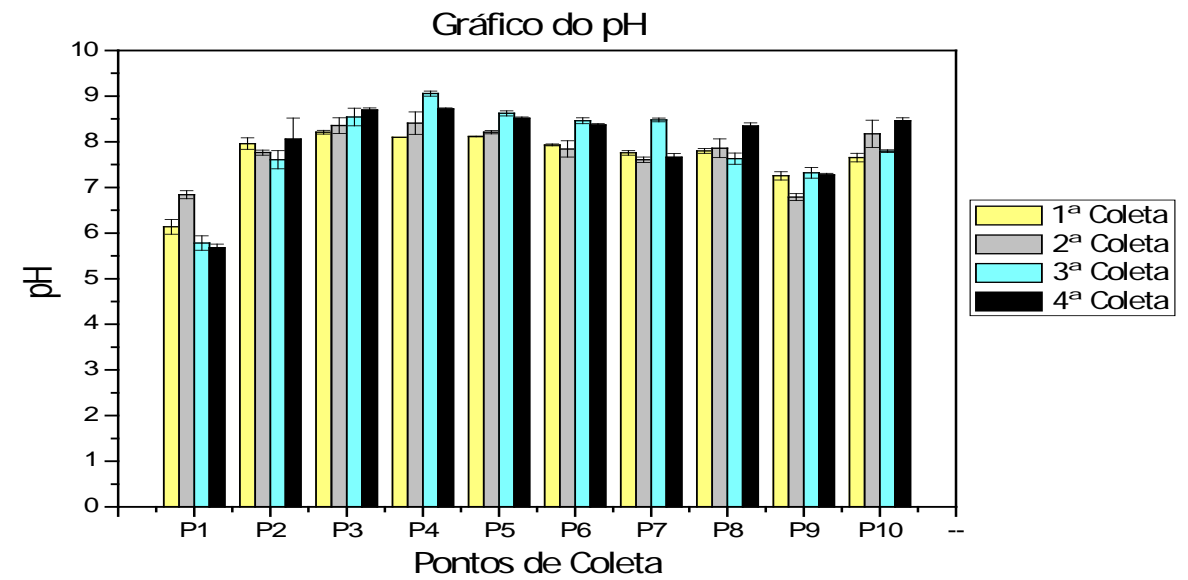

Gráfico 03: pH.

Nas analises constatou-se que o $\mathrm{pH}$ dos solos analisados são básicos, pois os pontos apresentaram $\mathrm{pH}$ maiores que 7 , geralmente entre 8 e 10, com exceção do ponto 1 que possui $\mathrm{pH}$ entre 5,5 e 7.

Provavelmente nestes solos os sais se hidrolisaram obtendo-se meios básicos, com $\mathrm{pH}$ maiores que 7, sendo assim um ponto favorável para os postes de liga metálica contendo ferro, pois os sais, neste caso, podem agir como inibidores de corrosão, passivando o ferro (GENTIL, 2011).

A matéria orgânica pode contribuir no processo corrosivo, devido à decomposição dos materiais, por microrganismos, tendendo a formar ácidos húmicos e fúlvicos. O Gráfico 04 é referente aos dados da matéria orgânica.

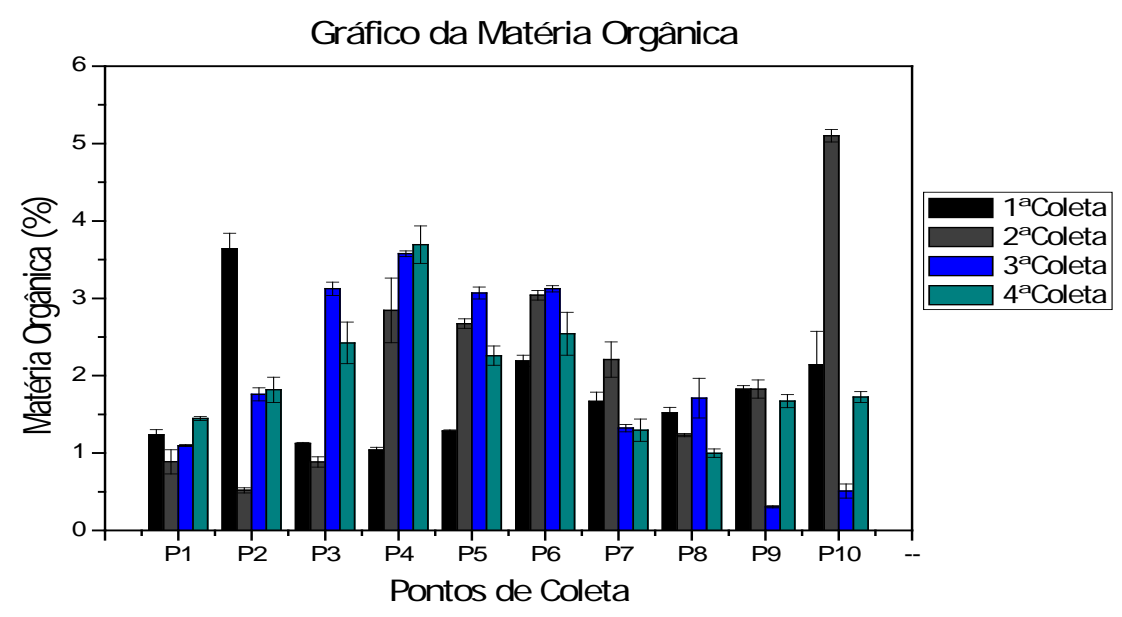

Gráfico 04: Matéria Orgânica.

Os pontos apresentaram baixa porcentagem da matéria orgânica, na maioria das vezes valores entre $1 \%$ e $5,5 \%$, não sendo assim um fator tão agravante para o processo corrosivo, mas que pode contribuir.

A razão de adsorção de sódio (RAS) pode ser considerada como um importante parâmetro para saber como o solo se classifica segundo sua salinidade. Os valores da salinidade foram calculados em função de teores de $\mathrm{Na}$, $\mathrm{Ca}$ e $\mathrm{Mg}^{+}$, obtidos pela equação 3:

$$
\mathrm{RAS}=\mathrm{Na} /\left[1 \frac{1}{2} \mathrm{x}(\mathrm{Ca}+\mathrm{Mg})\right]^{1 / 2}
$$

RAS = Razão de Adsorção de Sódio

$\mathrm{Na}=$ Concentração de sódio em $\mathrm{cmol}_{\mathrm{C}} / \mathrm{l}$

$\mathrm{Ca}=$ Concentração de cálcio em $\mathrm{cmol}_{\mathrm{c}} / \mathrm{l}$

$\mathrm{Mg}^{+}=$Concentração de magnésio em $\mathrm{cmol}_{\mathrm{C}} / \mathrm{l}$ 
Tabela 02- Resultados do RAS.

\begin{tabular}{llllllllllll}
\hline \multirow{4}{*}{ RAS } & Coletas & P1 & P2 & P3 & P4 & P5 & P6 & P7 & P8 & P9 & P10 \\
& $1^{\text {a }}$ & 0,09 & 0,26 & 0,34 & 0,41 & 0,05 & 0,12 & 0,02 & 0,02 & 0,04 & 0,05 \\
& $2^{\text {a }}$ & 0,08 & 0,00 & 0,18 & 1,20 & 0,03 & 0,02 & 0,00 & 0,00 & 0,00 & 0,00 \\
& $3^{\text {a }}$ & 0,02 & 0,01 & 0,26 & 1,79 & 0,11 & 0,05 & 0,02 & 0,00 & 0,00 & 0,00 \\
& $4^{\text {a }}$ & 0,29 & 0,08 & 0,08 & 1,13 & 0,19 & 0,07 & 0,07 & 0,00 & 0,00 & 0,02 \\
\hline
\end{tabular}

O Laboratório de Salinidade dos Estados Unidos (Universidade da Califórnia - Riverside) classifica os solos quanto à salinidade em função da condutividade elétrica do extrato de saturação (CE), da percentagem de sódio trocável (PST) ou da relação de adsorção de sódio (RAS) e do pH. Baseada nesse parâmetro tem-se a tabela 03.

Tabela 03- Classificação dos solos quanto à salinidade baseada na dos (EUA).

\begin{tabular}{llll}
\hline SOLO & CE(mmhos.cm-1 & RAS(\%) & $\mathrm{pH}$ \\
\hline Normal & $<4$ & $<13$ & $<8,5$ \\
Salino & $>4$ & $<13$ & $<8,5$ \\
Sódico & $<4$ & $>13$ & $\geq 8,5$ \\
Salino/Sódico & $>4$ & $>13$ & $<8,5$ \\
\hline
\end{tabular}

Fazendo o comparativo verificou-se que todos os solos analisados apresentam caráter salino, pois possuem CE maior que 4, RAS menor que 13 e na maioria das analises o $\mathrm{pH}$ dos solos foi menor que 8,5 .

O solo sendo salino, implica dizer que existe um acumulo de sais na superfície do solo, esse acumulo de sais influencia na velocidade e intensidade da ação corrosiva, o que torna o solo mais agressivo (GENTIL, 2011).

\section{CONCLUSÃO}

Com base nos resultados obtidos nesse trabalho, pode-se verificar que, com exceção do ponto 4, o teor de umidade presente nos solos é baixo e em épocas não chuvosa a umidade tende a se tornar menor nos solos e com isso vem diminuindo a influencia da umidade no processo corrosivo. Constatou-se ainda que a condutividade vai aumentando na medida que o teor de umidade vai diminuindo, possivelmente por causa da menor solubilização dos sais, então, na medida que um agente corrosivo vai diminuindo o outro tende a aumentar.

$\mathrm{O}$ pH do solo estudado não é um fator que oferece grande risco na corrosão de estruturas de ferro, pois verificou-se nos resultados $\mathrm{pH}$ maiores que 7 , com exceção do ponto 1 . Desta forma, o ferro tende a se passivar, ou seja, esse meio começara a servir como um inibidor de corrosão. Além disso, a matéria orgânica influencia através dos ácidos húmicos e fúlvicos, consequentemente pode diminuir o valor do $\mathrm{pH}$, entretanto os valores de matéria orgânica ficaram abaixo de 6\%, representando assim uma influencia muito pequeno na corrosão.

O fator mais expressivo além da umidade é a salinidade, obtido os resultados dos íons cálcio, magnésio e sódio, foi possível calcular a razão de adsorção do sódio e junto com a condutividade e o pH caracterizou os solos sendo do tipo salino, o que contribui para aceleração do processo corrosivo em conjunto dos outros agentes, como a água, matéria orgânica entre outros.

O conhecimento do solo como meio corrosivo é de extrema importância para poder-se avaliar a intensidade do processo corrosivo, avaliar quais componentes são mais agressivos para determinados tipos de metais e desta forma ser possível tomar medidas preventivas contra o problema. Com intuito de tardar esse processo corrosivo ou preveni-lo possíveis soluções seriam a utilização de pequenas peças de zinco colocadas entre o parafuso e a base dos postes, proteção catódica por metal de sacrífico, fazer revestimentos simples que já são empregados como a pintura, só que não apenas com a finalidade estética mais buscando proteção, usando tintas anticorrosivas como o zarcão e até mesmo usar as duas técnicas em conjunto. 


\section{Analysis of Soil Composition and its Influence on Corrosive Process Metallic Poles at UFERSA, Campus Caraúbas-RN.}

Abstract: Among the materials most widely used in the construction and assembly of poles and towers stand out metals, which when exposed to the environment are subject to corrosion. Corrosion may be defined as a process of decay of metal materials and non-metallic influenced by physical and chemical agents linked or not to mechanical stress. Agents that influence the corrosion process are: soil, temperature, pollution, humidity and gases. The soil has a large number of variables that contribute to the increase of the corrosion process in metallic structures, some were studied in this work following the manual for soil analysis methods of the Brazilian Agricultural Research Corporation (EMBRAPA). The results were based according to physical and chemical analysis of moisture, conductivity, $\mathrm{pH}$, organic matter, magnesium, sodium and calcium. It is possible with these results characterize the soil according to its salinity, showing that soils are saline type. It is necessary this work for a study on the influence of physical and chemical composition of the soil corrosivity of the lampposts of UFERSA-Campus Caraúbas-RN, trying to propose possible technical prevention of the corrosion process, such as cathodic protection for metal of sacrifice and coat with anti-corrosive paint.

Keywords: Corrosion; metal structures; soil; physical and chemical analyzes; prevention techniques

\section{REFERÊNCIAS BI BLIOGRÁFI CAS}

AMARAL, Cristina Torres; CORREIA, Martineli Priscila e PEREIRA, Paulo Tibúrcio. Corrosão em estruturas metálicas: uma breve discussão acerca da prevenção em torres de telecomunicações. Departamento de Ciências Exatas e Tecnologia - Engenharia de Telecomunicações 2008.

ALVES, Joaquim de Lima Junior; LUIZ, André Pereira da Silva. Estudo do processo de salinização para indicar medidas de prevenção de solos salinos. Centro Científico Conhecer - Goiânia, vol.6, N.11; 2010.

BRUSAMARELLO, V.J., HUDSON, J.L., SCULLY, J.R., LUNT, T., MIKHAILOV, A.S, "Spatial interactions among localized corrosion sites”, Journal of Electrochemical Society, v. 149, n. 5, 2002. DUARTE, M. Meio ambiente no século 21. Rio de Janeiro: Sextante, p. 245-257, 2003.

EMBRAPA. Centro nacional de pesquisa de solos. Manual de métodos de análise de solos. 2 ed. rev. e atual. Rio de Janeiro: Embrapa, 1997.

FRAUCHES-SANTOS, C.; ALBUQUERQUE, M. A.; OLIVEIRA, M. C. C.; Echevarria, A. A Corrosão e os Agentes Anticorrosivos. Rev. Virtual Química, 2014.

GETIL, Vicente. Corrosão. Editora: LGT, 6 edição, Rio de Janeiro,2011.

NÓBREGA, Carlos Alberto; CHANG, Hung Kiang; BRAGA, Antônio Celso de Oliveira; FERREIRA, Josely; OLIVA, Andreza e PEDE, Marco Aurélio Zequim. Avaliação preliminar da corrosividade de solo com o emprego de resistividade elétrica em uma planta industrial utilizada para armazenamento de derivados de petróleo. Geociências, v. 22, N. Especial, 2003.

NEVES, Sabrina da Silva. Estudo da corrosão do solo de aços para dutos protegidos. Programa de Pós-Graduação em Engenharia de Minas, Metalúrgica e Materiais (PPGEM) - Universidade Federal do Rio Grande do Sul, pg 1-6, 2007.

MAGALHÃES, Fátima Cristina; BATISTA, Walmar; OLIVEIRA, Mônica de Penna; DOMINGOS, Edilson da Silva; BARRETO, Herval de Oliveira e CARLOS, José Moreira da Costa. Critérios para avaliação da corrosividade de solos por bactérias redutoras de sulfato, In: $6^{\circ}$ Conferência sobre Tecnologia de Equipamentos (COTEQ), Salvador - Brasil, 2002.

PEIXOTO, Daniel D.D. Produção de imagem aérea ortorretificada da UFERSA Campus Caraúbas através de VANT. 2015. 36 pg. Trabalho de conclusão de curso. Bacharelado em Ciência e Tecnologia (UFERSA, Campus Caraúbas, 2015).

SILVA, José M. Influencia da agressividade do solo na corrosão das linhas de transmissão do sistema elétrico paraguaio. Salvador, 9 $^{\text {a }}$ Conteq, 2007. 